\title{
ORIGINAL
}

\section{Restrictive fluid management versus usual care in acute kidney injury (REVERSE-AKI): a pilot randomized controlled feasibility trial}

\author{
Suvi T. Vaara ${ }^{1,2,3^{*}}$ (D), Marlies Ostermann ${ }^{4}$, Laurent Bitker ${ }^{2,5}$, Antoine Schneider ${ }^{6}$, Elettra Poli ${ }^{6}$, Eric Hoste ${ }^{7}$, \\ Jan Fierens ${ }^{7}$, Michael Joannidis ${ }^{8}$, Alexander Zarbock ${ }^{9}$, Frank van Haren ${ }^{10,11}$, John Prowle ${ }^{12}$, Tuomas Selander $^{13}$, \\ Minna Bäcklund', Ville Pettilä ${ }^{1}$, Rinaldo Bellomo ${ }^{2,14}$ on behalf of the REVERSE-AKI study team
}

(ㄱ) 2021 The Author(s)

\begin{abstract}
Purpose: We compared a restrictive fluid management strategy to usual care among critically ill patients with acute kidney injury (AKI) who had received initial fluid resuscitation.

Methods: This multicenter feasibility trial randomized $100 \mathrm{AKI}$ patients 1:1 in seven ICUs in Europe and Australia. Restrictive fluid management included targeting negative or neutral daily fluid balance by minimizing fluid input and/or enhancing urine output with diuretics administered at the discretion of the clinician. Fluid boluses were administered as clinically indicated. The primary endpoint was cumulative fluid balance $72 \mathrm{~h}$ from randomization.

Results: Mean (SD) cumulative fluid balance at $72 \mathrm{~h}$ from randomization was $-1080 \mathrm{~mL}$ (2003 $\mathrm{mL}$ ) in the restrictive fluid management arm and $61 \mathrm{~mL}(3131 \mathrm{~mL})$ in the usual care arm, mean difference $(95 \% \mathrm{Cl})-1148 \mathrm{~mL}(-2200$ to -96$) \mathrm{mL}, P=0.033$. Median [IQR] duration of AKI was 2 [1-3] and 3 [2-7] days, respectively (median difference -1.0 $[-3.0$ to 0.0$], P=0.071)$. Altogether, 6 out of $46(13 \%)$ patients in the restrictive fluid management arm and 15 out of 50 (30\%) in the usual care arm received renal replacement therapy (RR 0.42; 95\% Cl 0.16-0.91), $P=0.043$. Cumulative fluid balance at $24 \mathrm{~h}$ and 7 days was lower in the restrictive fluid management arm. The dose of diuretics was not different between the groups. Adverse events occurred more frequently in the usual care arm.
\end{abstract}

Conclusions: In critically ill patients with AKI, a restrictive fluid management regimen resulted in lower cumulative fluid balance and less adverse events compared to usual care. Larger trials of this intervention are justified.

Keywords: Acute kidney injury, Critically ill, Fluid balance, Restrictive fluid management

\footnotetext{
*Correspondence: suvi.vaara@helsinki.fi

${ }^{3}$ Intensive Care Unit M1, Meilahti Hospital, Box 340, 00290 Helsinki, Finland

Full author information is available at the end of the article
}

The members of the The REVERSE-AKI study team are listed in the acknowledgements section.

\section{Introduction}

Many critically ill patients develop acute kidney injury (AKI). AKI is associated with an increased risk of adverse outcomes $[1,2]$. Current management of AKI remains supportive and includes optimization of hemodynamics and fluid status, avoidance of nephrotoxins [3] and, in severe cases, renal replacement therapy (RRT) $[1,2]$. Novel therapies are being evaluated in cardiac surgical [4] and septic patients [5], but no specific pharmacological 
therapies for general intensive care unit (ICU) patients are currently available.

While fluid therapy is the traditional cornerstone of AKI prevention and treatment [6], patients with AKI are also especially prone to develop fluid overload [7]. Such fluid overload is associated with increased mortality [7-11] and progression of AKI $[12,13]$. Thus, a more restrictive fluid management approach among adequately resuscitated patients with AKI might improve outcomes by reducing congestion and improving kidney and other organ function. Notably, a feasibility trial in septic shock patients comparing restrictive fluid resuscitation versus usual care reported a lower risk of worsening of AKI in the restrictive group [14].

Trials among general ICU patients [15], or those with sepsis $[14,16]$ or acute respiratory distress syndrome (ARDS) [17] have found various fluid restrictive strategies to be safe. Among patients undergoing major abdominal surgery, however, restrictive intra- and post-operative fluid resuscitation and management compared to usual care increased the risk of AKI [18]. Thus, research investigating the role of restrictive fluid management specifically in critically ill patients with AKI has been identified as a research priority $[3,19,20]$. To date, such trials have not been performed.

Accordingly, we conducted a multinational, unblinded, randomized, controlled, feasibility pilot trial comparing a restrictive fluid management (RFM) regimen to usual care among ICU patients with AKI to study the feasibility of such intervention in terms of separation, safety, and compliance. We tested the hypothesis that the proposed RFM regimen would lead to a lower cumulative fluid balance at $72 \mathrm{~h}$ post-randomization.

\section{Methods}

\section{Trial design}

We conducted an investigator-initiated, multicenter, unblinded, randomized, controlled, parallel pilot feasibility study (clinicaltrials.gov identifier NCT03251131) in five European and two Australian ICUs. The trial protocol and statistical analysis plan were published prior to completion of data collection [21]. In each participating site, the trial was approved by the relevant research ethics committee prior to commencing screening. Depending on local practices and ethics approval, we obtained written informed consent either prior to enrollment from patient or proxy, or alternatively a deferred consent with written informed consent obtained from patient or proxy as soon as possible. If consent was not granted or withdrawn, approval of using data collected that far was sought. Trial was performed according to the Declaration of Helsinki and its later amendments.

\section{Take-home message}

Among critically ill patients with acute kidney injury, a fluid restrictive management regimen as compared to usual care led to a lower cumulative fluid balance after $72 \mathrm{~h}$ from randomization. Adverse events were more frequent in the usual care arm.

We randomized patients to either RFM or usual care using an electronic platform with allocation ratio of 1:1. Severity of AKI (stage 1 vs stage 2 or 3 according to the Kidney Diseases: Improving Global Outcomes (KDIGO) criteria [3]) and presence of clinical signs of fluid accumulation (defined by peripheral pitting edema and/or positive fluid balance with $\mathrm{P} / \mathrm{F}$ ratio less than $200 \mathrm{mmHg}$ ) served as stratification variables. We used permuted blocks of varying size (2-4). Due to the nature of intervention, only the statistician conducting the data analysis remained blinded to treatment allocation.

\section{Patients}

We included patients who (1) were 18 years or older and admitted to critical care with an arterial line in place, (2) were in critical care for at least 12 but no more than $72 \mathrm{~h}$ (3) had AKI but were not receiving RRT, (4) were judged by the treating clinician not to be hypovolemic, and (5) were likely to remain in critical care for $48 \mathrm{~h}$ after randomization.

We defined AKI according to the following criteria: (1) increase in plasma creatinine 1.5 times or more above baseline without a decline of $27 \mathrm{umol} / \mathrm{L}$ or more from the last preceding measurement and/or (2) overall urine output less than $0.5 \mathrm{ml} / \mathrm{kg} / \mathrm{h}$ (or $6 \mathrm{ml} / \mathrm{kg}$ ) for the previous $12 \mathrm{~h}$. Exclusion criteria are listed in Fig. 1. The Electronic Supplementary Material (ESM) Tables S1 and S2 present detailed definitions of the eligibility criteria.

\section{Interventions}

The trial intervention (RFM) consisted of a bundle of treatment recommendations for clinicians treating the trial patients. The overall aim was to reach negative fluid balance on the days following randomization. These recommendations included, first, restricting total daily fluid input only to medications and nutritional fluids (enteral or parenteral) and blood products (if clinically necessary). Second, use of maintenance intravenous fluids was permitted only if enteral nutrition was not tolerated and parenteral nutrition contraindicated. Third, fluid bolus therapy could be given as clinically deemed necessary. Fourth, matching fluid output (with unrestricted use of diuretics) to fluid input whenever possible to achieve a preferably negative cumulative fluid balance but always 


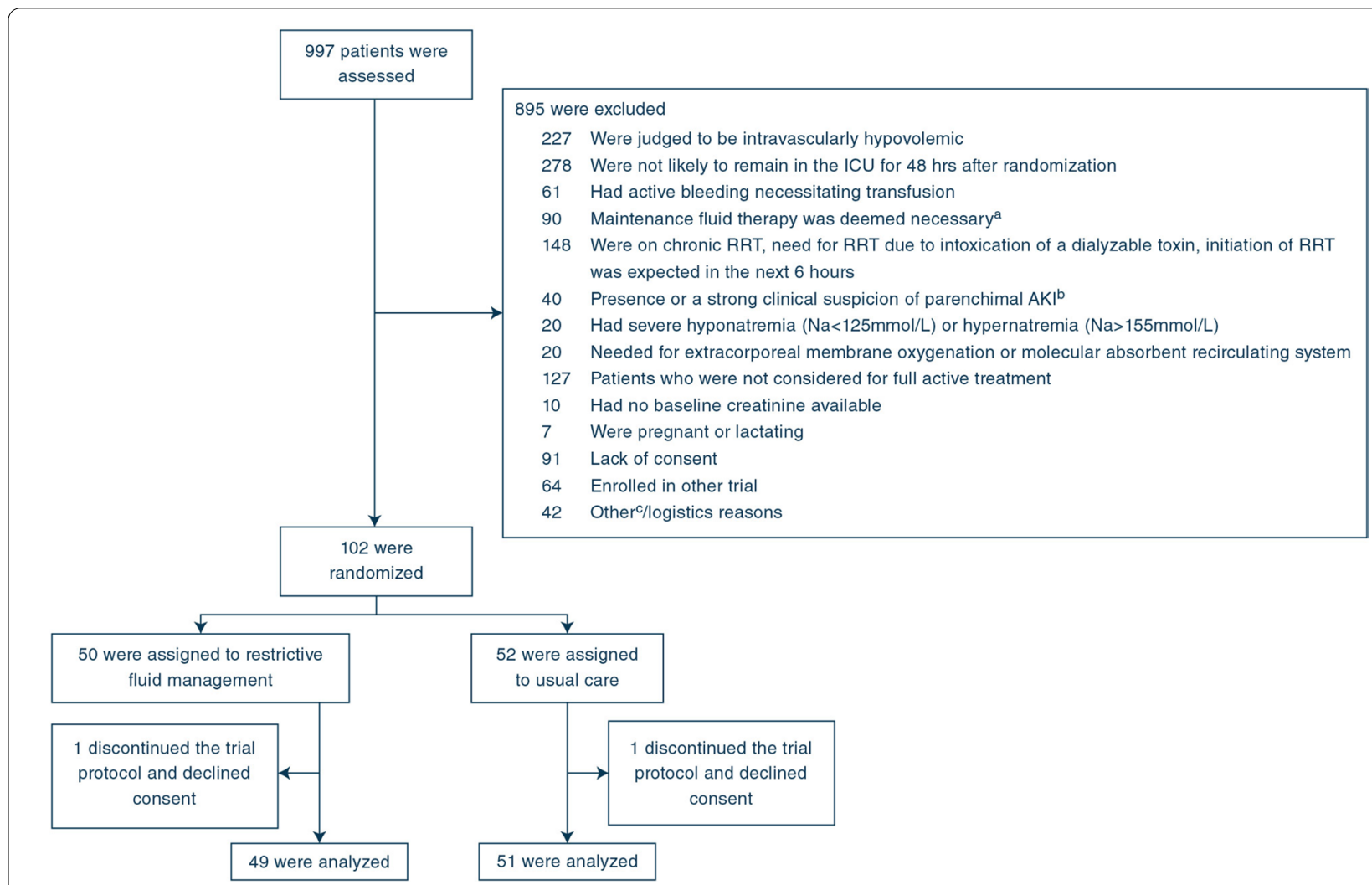

Fig. 1 Flowchart of trial patients. ICU intensive care unit, RRT renal replacement therapy. ${ }^{\text {a }}$ Diabetic ketoacidosis, non-ketotic coma, severe burns or other clinical reason determined by the medical staff. ${ }^{b}$ Glomerulonephritis, vasculitis, acute interstitial nephritis, or post-renal obstruction. ${ }^{c}$ Including two exclusion criteria that were removed in a protocol amendment in April 2018 (1) Metformin-induced lactic acidosis or acute liver failure $(n=8)(2)$ AKI stage 2 or greater is known to have been present for $>48 \mathrm{~h}(n=14)$. These criteria were amended to clarify the exclusion criteria

less than $300 \mathrm{~mL} /$ day. Fifth, if the fluid balance target could not be achieved by such means, consideration of RRT to remove the required fluid. Commencing RRT was not mandated in the trial and, if RRT was not considered clinically desirable, the protocol indicated acceptance of a less than targeted fluid balance temporarily (for $24 \mathrm{~h}$ ) up to $900 \mathrm{~mL}$.

In the usual care group, fluid management was at the discretion of the treating clinical team.

Fluid balance was calculated by subtracting total fluid output (urine output, losses to drains, losses from gastrointestinal tract, ultrafiltration by RRT) from total fluid input (intravenous and oral). Insensible losses were not considered. The intervention period was 7 days from randomization or until ICU discharge whatever occurred first.

\section{Outcomes}

The primary outcome was cumulative fluid balance at $72 \mathrm{~h}$ after randomization adjusted for stratification variables $[21,22]$. Secondary outcomes included duration of AKI in days defined by the KDIGO creatinine and urine output criteria (truncated at ICU discharge or 14 days whichever came first), number of patients requiring RRT (truncated at 14 days, including initiation of RRT after ICU discharge), cumulative fluid balance at $24 \mathrm{~h}$ after randomization and at ICU discharge (or truncated at 7 days if ICU stay exceeded 7 days), and cumulative dose of diuretics (furosemide) during the intervention period (while in the ICU, maximum of 7 days) adjusted for the duration of the observation period.

Exploratory outcomes included days free of mechanical ventilation and alive at 14 days, days free of vasopressors and alive at 14 days, days free of ICU and alive at 14 days, days free of RRT and alive (assessed at 90 days), 90-days dialysis dependence and 90-days mortality. The safety and feasibility outcomes included the number of patients with one or more (serious) adverse events (AE) and reactions in both arms (detailed definitions are provided in Table S3), screened versus recruited patients ratio, recruitment rate (patients/ center/month), and protocol compliance (number of patients with protocol violations in both arms). 


\section{Statistical analysis}

Details of statistical analysis plan have been published [21]. We estimated that 50 patients per arm would be required to show a difference of $1200 \mathrm{~mL}$ in the primary outcome between treatment arms with a two-sided alpha of 0.05 and power exceeding $80 \%$. Based on unpublished data among AKI patients in the FINNAKI study [1], we estimated that cumulative fluid balance at $72 \mathrm{~h}$ would be $2700 \mathrm{~mL}$ in the usual care group, and thus $1500 \mathrm{~mL}$ in the fluid restrictive group (both with a SD of $2000 \mathrm{~mL}$ ).

We performed the primary analysis on the intentionto-treat (ITT) population defined as all randomized subjects with consent to use data in the analysis. An additional sensitivity analysis was conducted in the per-protocol population, defined as the ITT population after exclusion of subjects who experienced protocol violation(s) or stayed in the ICU for less than $48 \mathrm{~h}$ post-randomization.

The primary, secondary, and exploratory outcomes were adjusted for the stratification variables [22] (mild vs severe AKI and presence/absence of clinical signs of fluid accumulation) using two-tailed logistic regression (dichotomous outcomes) or a linear model (continuous outcome variables, mean or median regression model). We report risk ratios (RR) with $95 \%$ confidence intervals (CIs) or difference in means/median with $95 \%$ CIs.

We used Shapiro-Wilk test for continuous variables to assess assumption of normality. We set the statistical significance to 0.05 and report two-sided $P$ values. Analyses were conducted using $\mathrm{R}$ statistical software version 3.6.2 and SPSS 24.

\section{Results}

\section{Patients}

Between October 2017 and January 2020, we screened 997 patients and randomized 102 patients. One patient in each group declined consent leaving 100 patients for analysis. Randomization occurred at a median [IQR] 30.7 [20.4-46.2] hours after ICU admission. Fortynine patients (49\%) were assigned to the RFM arm and 51 (51\%) to the usual care arm. Figure 1 presents the patient flow. Further, two patients in the RFM arm and three patients in usual care arm withdrew consent and their data obtained until withdrawal were included in the analysis.

Patient baseline characteristics and status at randomization were well-balanced (Table 1). The most frequent reasons for ICU admission were cardiac arrest (8.2\%), septic shock (7.2\%), and gastrointestinal perforation/rupture (7.2\%). Suspected etiology of AKI was multifactorial in $55(55 \%)$ patients. Table S5 presents the observed AKI risk factors.

\section{Primary, secondary, and exploratory outcomes}

At 72 h, 66 (66\%) patients were still in ICU, while 29 (29\%) were discharged, four were deceased (one in RFM arm and three in usual care), and one had withdrawn consent for further data collection. The median length of stay from randomization to discharge among these 34 patients was 49.2 [32.6-51.9] in RFM arm and 51.0 [29.3$60.2]$ hours in the usual care arm. The primary outcome was assessed after a median [IQR] 72.0 [53.9-72] hours from randomization. Figure 2 presents fluid input and output data along with daily fluid balance in both arms. Table 2 presents the primary and secondary outcomes. The mean difference $(95 \% \mathrm{CI})$ in cumulative fluid balance at $72 \mathrm{~h}$ was $-1148(-2200$ to -96$) \mathrm{mL}, P=0.033$. There was no difference in duration of AKI but fewer patients in the RFM group received RRT compared to the usual care group. Two additional patients in the usual care arm commenced RRT after day 14. Cumulative fluid balance at $24 \mathrm{~h}$ and at ICU discharge or on day 7 were significantly lower in the RFM group. Diuretics were administered to $35(71.4 \%)$ in the RFM group and $38(74.5 \%)$ in the usual care group. The median [IQR] day number of first administration of diuretics was 1 [1] in both groups. There were no differences in the cumulative doses of diuretics adjusted for observation days. The crude primary outcome was in line with the adjusted analysis (Table S6). There were no significant differences in the adjusted or unadjusted exploratory outcomes (adjusted in Table 3, crude in Table S7). Figure S1 presents the daily SOFA score according to treatment allocation.

\section{Safety and feasibility outcomes}

Eleven (22.4\%) patients in the RFM group and 25 (49\%) in the usual care group experienced one or more $\mathrm{AE}$ (risk ratio 0.46 ; $95 \% \mathrm{CI} 0.36-0.63, P=0.001)$. Serious adverse events (SAE) were observed in six (12.2\%) patients randomized to RFM group and in $16(31.4 \%)$ randomized to usual care group (RR 0.39; 95\% CI 0.15-0.86, $P=0.031$ ). Table S8 provides a detailed description of AEs and SAEs. Most events were related to disturbances in serum electrolyte concentrations and RRT. The median [IQR] number of patients recruited per center/month was 0.8 [0.5-1.7] (Figure S2).

\section{Fluid protocol and protocol violations}

Protocol violations occurred in 18 (36.7\%) in the RFM arm and in $5(9.8 \%)$ in the usual care arm. In the RFM arm, use of excess maintenance fluid was the most frequently observed protocol violation in $9(18.4 \%)$ patients (Table S9). Moreover, five patients in the RFM arm had a protocol suspension for the following reasons: hypernatremia $(n=2)$, need for surgery $(n=1)$, complete heart block (not considered to be attributable to study 
Table 1 Patient characteristics

\begin{tabular}{|c|c|c|}
\hline Characteristic & $\begin{array}{l}\text { Restrictive fluid management } \\
(n=49)\end{array}$ & $\begin{array}{l}\text { Usual care } \\
(n=51)\end{array}$ \\
\hline Age, median $[I Q R]$, years & $71[60-77]$ & $64.5[56.2-74]$ \\
\hline Male sex, $n(\%)$ & $30(62.5)$ & $36(70.6)$ \\
\hline Body mass index, median [IQR], kg/m² & $25.6[22.8-29.5]$ & $26.4[22.7-31.1]$ \\
\hline Hypertension, $n(\%)$ & $26(54.2)$ & $32(65.3)$ \\
\hline Diabetes, $n(\%)$ & $24(50)$ & $33(66)$ \\
\hline Chronic heart failure, $n(\%)$ & $6(12.5)$ & $7(14)$ \\
\hline Coronary artery disease, $n$ (\%) & $9(18.8)$ & $16(31.4)$ \\
\hline Chronic obstructive pulmonary disease, $n(\%)$ & $8(16.7)$ & $14(27.5)$ \\
\hline Chronic kidney disease, $n(\%)$ & $5(10.6)$ & $6(11.8)$ \\
\hline Chronic liver disease, $n(\%)$ & $4(8.3)$ & $3(5.9)$ \\
\hline Surgical ICU admission, $n$ (\%) & $22(44.9)$ & $20(40)$ \\
\hline Emergency ICU admission, $n(\%)$ & $37(75.5)$ & $41(82)$ \\
\hline Time from hospital admission to ICU admission, median [IQR], days & $1(0.1-4)$ & $0.9(0.2-2.9)$ \\
\hline Time from ICU admission to randomization, median [IQR], h & $33.3(21.4-45.9)$ & $30(20.3-46)$ \\
\hline \multicolumn{3}{|l|}{ Data at randomization } \\
\hline \multicolumn{3}{|l|}{ AKI inclusion criteria } \\
\hline Creatinine & $25(51)$ & $28(54.9)$ \\
\hline Urine output & $10(20.4)$ & $8(15.7)$ \\
\hline Creatinine and urine output & $14(28.6)$ & $15(29.4)$ \\
\hline SAPS II score, median [IQR] ${ }^{a}$ & $41.5[31.3-51.5]$ & $43[30-50]$ \\
\hline SOFA score, median $[I Q R]^{b}$ & $9[8-11]$ & $9[7-11]$ \\
\hline AKI stage 2 to 3 (stratification), $n$ (\%) & $31(63.3)$ & $33(64.7)$ \\
\hline Fluid overload present (stratification), $n(\%)^{c}$ & $21(42.9)$ & $22(43.1)$ \\
\hline Respiratory supportmechanical ventilation, $n(\%)$ & $24(49)$ & $33(66)$ \\
\hline Vasoactives, $n(\%)$ & $30(61.2)$ & $37(72.5)$ \\
\hline Sepsis, $n(\%)$ & $27(55.1)$ & $30(58.8)$ \\
\hline Septic shock, $n(\%)$ & $5(10.2)$ & $9(17.7)$ \\
\hline Cumulative fluid balance from ICU admission to randomization, mean (SD), $\mathrm{mL}$ & $2437(2103)$ & $1982(2434)$ \\
\hline
\end{tabular}

The number of missing data is presented in Table S4 in the Electronic Supplementary Material

AKI acute kidney injury, SAPS simplified acute physiology score, SOFA sequential organ failure assessment

a Data of one or several SAPS II score subcomponents were missing from 11 patients in the RFM group and 15 in the usual care group. Their scores are omitted here

b The hepatic subcomponent score was assumed to be 0 in those 4 in RFM group and 3 in the control group with missing bilirubin values

c Definition of the stratification variable: presence of peripheral pitting edema and/or positive fluid balance with $\mathrm{P} / \mathrm{F}$ ratio less than $200 \mathrm{mmHg}$

intervention) $(n=1)$, and withdrawal of consent $(n=1)$. One patient in the usual care arm had protocol suspension as overall ICU care was withdrawn.

\section{Per-protocol analysis}

Twenty-three patients (46.9\% of 49 ) in the RFM arm and $37(72.5 \%$ of 51$)$ in the usual care arm were included in the per-protocol analysis. The primary or secondary outcome variables were not different in the per-protocol population except for fluid balance at $24 \mathrm{~h}$ (Table S10).

\section{Discussion}

\section{Key findings}

In this multicenter, multinational, unblinded, randomized, controlled, feasibility pilot study involving 100 critically ill patients with AKI who were considered adequately resuscitated, we assessed whether, compared to usual care, a restrictive fluid management regimen would lead to a meaningful difference in cumulative fluid balance after $72 \mathrm{~h}$ from randomization without signals of harm. We found the RFM protocol to be effective in terms of achieving a lower cumulative fluid balance at $72 \mathrm{~h}$ compared to usual care. Moreover, patients in the RFM arm received RRT less frequently and had fewer adverse events than patients in the usual care arm.

\section{Relationship with previous studies}

No randomized controlled trial has studied a RFM regimen among critically ill patients with AKI. 


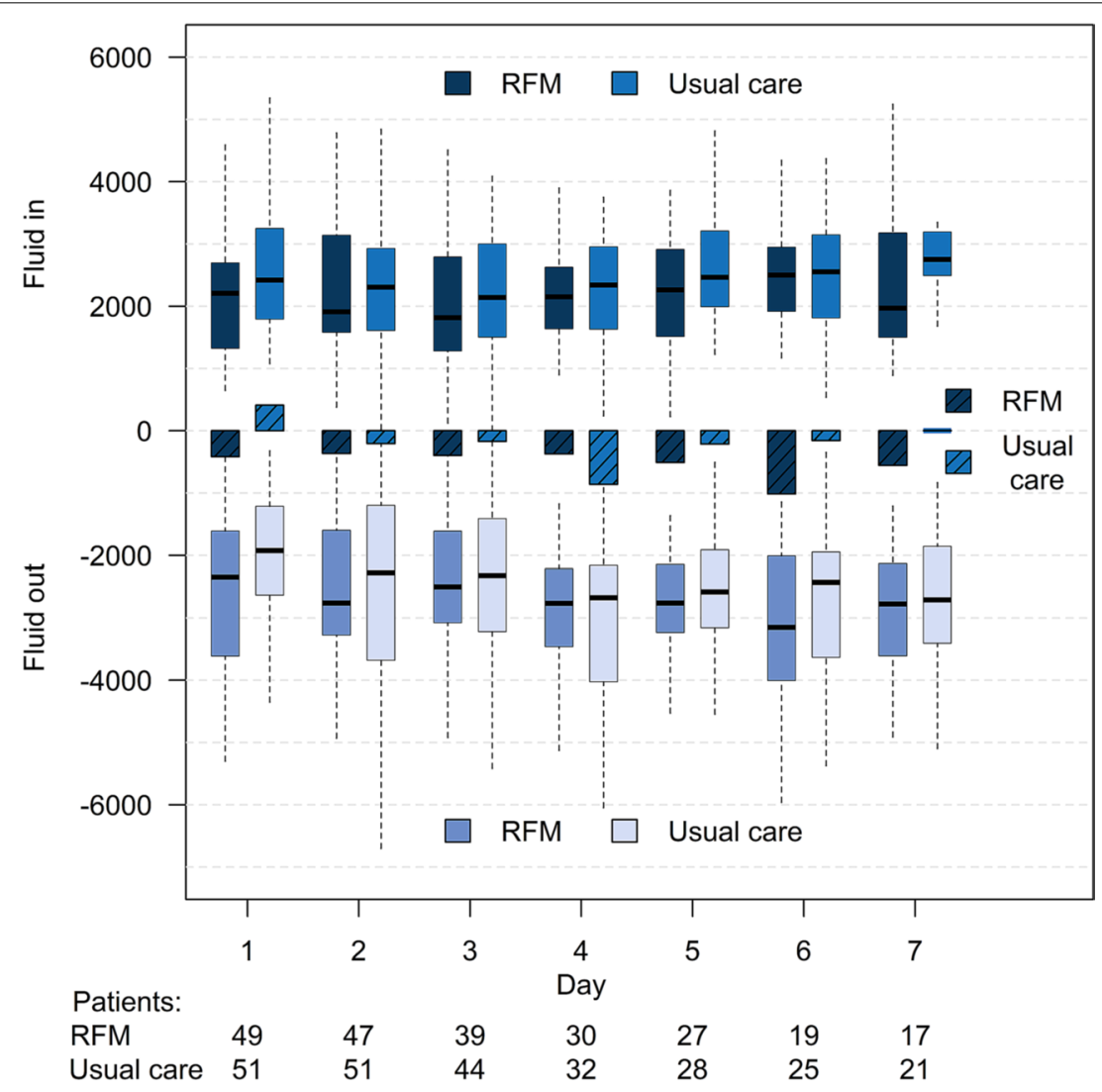

Fig. 2 Daily fluid input, fluid output and daily fluid balance from randomization in restrictive versus usual care. Boxplots represent median with IQR and range in $\mathrm{mL}$. Bars present the daily balance (not cumulative) in $\mathrm{mL}$. RFM restrictive fluid management

Table 2 Primary and secondary outcomes (adjusted for stratification variables)

\begin{tabular}{|c|c|c|c|c|}
\hline Outcome & $\begin{array}{l}\text { Restrictive fluid } \\
\text { management } \\
(n=49)\end{array}$ & $\begin{array}{l}\text { Usual care } \\
(n=51)\end{array}$ & $\begin{array}{l}\text { Restrictive fluid manage- } \\
\text { ment vs usual care }(95 \% \\
\mathrm{Cl}^{\mathrm{a}}\end{array}$ & $P$ value $^{b}$ \\
\hline $\begin{array}{l}\text { Cumulative fluid balance at } 72 \mathrm{~h} \text { from randomization, mean (SD) } \\
\mathrm{mL}^{c}\end{array}$ & $-1080(2003)$ & $61(3131)$ & $-1148(-2200 ;-97)$ & 0.033 \\
\hline Duration of AKI (days), median [IQR] ${ }^{\mathrm{d}}$ & $2[1-3]$ & $3[2-7]$ & $-1(-3 ; 0)$ & 0.071 \\
\hline Number of patients-receiving RRT, $n(\%)^{e}$ & $6 / 46(13)$ & $15 / 50(30)$ & $0.42(0.16 ; 0.91)$ & 0.043 \\
\hline $\begin{array}{l}\text { Cumulative fluid balance at } 24 \mathrm{~h} \text { from randomization, mean (SD) } \\
\mathrm{mL}^{\mathrm{c}}\end{array}$ & $-416(1194)$ & 409 (1566) & $-822(-1381 ;-264)$ & 0.004 \\
\hline Cumulative fluid balance at ICU discharge/day 7 , mean (SD) $\mathrm{mL}^{\mathrm{c}}$ & $-2166(2988)$ & $-650(4469)$ & $-1532(-3036 ;-29)$ & 0.046 \\
\hline Cumulative dose of furosemide per day, median [IQR] mg ${ }^{f}$ & $0(0-19)$ & $1.4(0-26.2)$ & $0(-11 ; 5.7)$ & 0.700 \\
\hline
\end{tabular}

$A K I$ acute kidney injury, $R R T$ renal replacement therapy

a Adjusted (severity of AKI and presence of fluid overload) difference in means/median or risk ratio with $95 \% \mathrm{Cls}$

b $P$ value derived from regression model adjusted for stratification variables (severity of AKI and presence of fluid accumulation)

c The last available value for cumulative balance was analyzed for all patients even if ICU discharge or consent withdrawal would have occurred before endpoint was fulfilled

d Truncated at 7 days, ICU discharge, or consent withdrawal. Data missing for three patients-receiving restricted fluid management and one in usual care

e Truncated at 14 days (RRT provided post-ICU discharge included)

${ }^{f}$ Per oral furosemide dose divided by 2 to make it comparable to intravenous doses 
Table 3 Exploratory outcomes (adjusted for stratification variables)

\begin{tabular}{|c|c|c|c|c|}
\hline Outcome & $\begin{array}{l}\text { Restrictive fluid } \\
\text { management }\end{array}$ & Usual care & $\begin{array}{l}\text { Restrictive fluid management } \\
\text { vs usual care }(95 \% \mathrm{Cl})^{\mathrm{a}}\end{array}$ & $P$ value $^{b}$ \\
\hline Days alive and free of mechanical ventilation, median $[\mathrm{IQR}]^{\mathrm{C}}$ & $13(9-14)$ & $11.5(1.5-14)$ & $0(-1 ; 6)$ & 0.284 \\
\hline Days alive and free of vasopressors, median [IQR ${ }^{c}$ & $12(10-14)$ & $11.5(7-13)$ & $1(0 ; 2)$ & 0.072 \\
\hline Days alive and free of ICU treatment, median $[\mathrm{IQR}]^{\mathrm{c}}$ & $8(3-11)$ & $2.5(0-11)$ & $2(0 ; 6)$ & 0.106 \\
\hline Days alive and free of RRT at 90 days, median $[\mathrm{IQR}]^{\mathrm{d}}$ & $90(85-90)$ & $90(33-90)$ & $0(0 ; 14)$ & 0.145 \\
\hline Dialysis dependence at 90-days, $n$ (\%) & $0 / 46(0)$ & $1 / 49(2)$ & $0.34(0.01 ; 8.55)$ & 1.000 \\
\hline 90-day mortality, n (\%) & $9 / 46(19.6)$ & $13 / 49(26.5)$ & $0.74(0.35 ; 1.46)$ & 0.387 \\
\hline
\end{tabular}

a Adjusted (severity of AKI and presence of fluid overload) difference in means/median with $95 \% \mathrm{Cls}$

b $P$ value derived from regression model adjusted for stratification variables (severity of acute kidney injury and presence of fluid accumulation)

c Truncated at 14 days or ICU discharge. Potential ICU readmissions not included. Data not available for two patients-receiving restrictive fluid management and for one receiving usual care

d Data not available for two patients in both groups

The FACTT trial, conducted from 2000 to 2005, compared conservative fluid management to liberal therapy in patients with ARDS and found no difference in 60-day mortality, but reported shorter duration of mechanical ventilation and a trend toward less RRT in the conservative arm [17]. Furthermore, a sub-analysis of patients with AKI found an association with high cumulative fluid balance and mortality [9]. In the FACTT, the cumulative fluid balance on day three (corresponding to the primary endpoint in the current analysis) was about $150 \mathrm{~mL}$ in the conservative arm and about $5000 \mathrm{~mL}$ in the liberal arm, both more positive than in our study [23]. Moreover, we observed a much lower actual fluid balance in the usual care group than we had assumed based on data collected in 2011-2012. Such a shift towards more restrictive fluid management practices among the critically ill has been reported also in another RFM pilot trial [24].

Most interventional fluid trials in the critically ill have concentrated on modifying fluid administration $[14,15$, 25-28] or enhancing fluid output with diuretics and/or ultrafiltration [29]. Our approach of combining the two sides of the coin in one trial gave the treating clinician more tools to individualize fluid therapy while still successfully targeting a clinically meaningful outcome measure. For example, the CLASSIC pilot trial among patients with septic shock achieved a difference in the administered resuscitation fluid but not in the total fluid balance [14]. Notably, fluids other than resuscitation fluid represent the largest proportion of the administered fluids [30, 31] even in patients with shock [32].

A dynamic intervention bundle such as ours may be more suitable for AKI patients who represent a heterogeneous group of patients with some having severely reduced urine output significantly affecting the achievable fluid output while others have a creatinine rise but preserved urine output. A French stepped wedge cluster
$\mathrm{RCT}$ is assessing another dynamic approach among general ICU patients [33]. The trial intervention involves fluid restriction and/or enhancement of fluid output to achieve a daily weight loss of $0.5 \mathrm{~kg}$ in patients with increasing daily weight [33]. However, no results are yet available. A pilot study among septic shock patients reported a rather large but non-significant difference between restrictive and usual care without any signals of harm, but the trial was stopped early as the predefined endpoint of a-500 $\mathrm{mL}$-difference in mean daily fluid balance was not met [24].

\section{Study implications}

Our results showed that, in ICU patients with AKI who are considered not to be hypovolemic, using a combined strategy targeting control of daily fluid balance is both feasible and safe. Adverse events were more frequent in the usual care arm, possibly related to more frequent use of RRT in this group. Moreover, the trial provides initial support to the hypothesis that fluid accumulation is linked to important outcomes among AKI patients such as need for RRT, a hypothesis that should be studied in larger clinical trials.

A quarter of excluded patients were ineligible due to being still considered intravascularly hypovolemic albeit having stayed in the ICU for at least $12 \mathrm{~h}$. This is an important patient cohort that should be considered when designing future RFM trials. Potentially, these patients could be enrolled, if the RFM protocol included a welldefined starting trigger after correction of hypovolemia such as using passive leg raising or other dynamic tests to assess fluid responsiveness [34, 35].

\section{Strengths and limitations}

Obvious strengths to our study include randomization to minimize selection bias and a multinational approach 
allowing simultaneous feasibility assessment in multiple locations with potentially different fluid treatment practices and increasing external validity. In addition, we used a treatment bundle targeting both fluid input and output to maximize the efficacy of the intervention, a dynamic two-pronged approach, which increased flexibility and allowed for individualization of treatment according to perceived patients' needs. Moreover, we collected data on multiple potential adverse events daily to be able to reliably identify any signal of harm potentially associated with RFM.

We acknowledge several limitations. First, blinding of the intervention was not possible due to its nature. Second, calculated fluid balance is not an exact measure, as measurement of fluid output in patients with high insensible losses (such as losses to wound dressing) is not exact. Thus, the actual fluid balance of the included patients in both groups may be lower than reported here. Third, we observed protocol violations in terms of use of excessive maintenance fluid. This may signal the presence of local practices [30,32] that need to be better addressed in future trials. Fourth, we excluded patients with treatment limitations albeit many from this heterogenous group could potentially have been enrolled. Nonetheless, we observed a signal for positive patient-centered outcomes in the frequency of RRT, which implies that fluid restriction may have an effect on clinical outcomes.

\section{Conclusions}

In a pilot multicenter, international, randomized, controlled trial of critically ill patients with AKI who were considered adequately fluid resuscitated, a restrictive fluid management regimen was feasible, resulted in fewer adverse events than usual care, and delivered a significant separation in fluid balance. The results also suggested benefit in terms of patient-centered outcomes implying that this intervention should now be studied in larger trials.

\section{Supplementary Information}

The online version contains supplementary material available at https://doi. org/10.1007/s00134-021-06401-6.

\footnotetext{
Author details

${ }^{1}$ Division of Intensive Care Medicine, Department of Anesthesiology, Intensive Care and Pain Medicine, University of Helsinki and Helsinki University Hospital, Helsinki, Finland. ${ }^{2}$ Department of Intensive Care, Austin Hospital, Melbourne, Australia. ${ }^{3}$ Intensive Care Unit M1, Meilahti Hospital, Box 340, 00290 Helsinki, Finland. ${ }^{4}$ Department of Critical Care, King's College London, Guy's \& St Thomas' Hospital, London, UK. ${ }^{5}$ CREATIS CNRS UMR5220 INSERM U1044 INSA-Lyon, Université de Lyon, Lyon, France. ${ }^{6}$ Adult Intensive Care Unit, Centre Hospitalier Universitaire Vaudois (CHUV), Lausanne, Switzerland. ${ }^{7}$ Intensive Care Unit, Ghent University Hospital, Ghent University, Ghent, Belgium. ${ }^{8}$ Division of Intensive Care and Emergency Medicine, Department of Internal Medicine, Medical University of Innsbruck, Innsbruck, Austria.

${ }^{9}$ Department of Anesthesiology, Intensive Care and Pain Medicine, University Hospital Münster, Münster, Germany. ${ }^{10}$ Australian National University Medical
}

School, Canberra, Australia. ${ }^{11}$ Intensive Care Unit, Canberra Hospital, Canberra, Australia. ${ }^{12}$ Critical Care and Preoperative Medicine Research Group, Centre for Translational Medicine and Therapeutics, William Harvey Research Institute, Queen Mary University of London, London, UK. ${ }^{13}$ Science Service Center, Kuopio University Hospital, Kuopio, Finland. ${ }^{14}$ Centre for Integrated Critical Care, School of Medicine, The University of Melbourne, Melbourne, Australia.

\section{Acknowledgements}

We warmly thank the patients and their families for participating. We are grateful to the clinical staff at participating ICUs for their contributions. The REVERSE-AKI study team: Austin Hospital: Rinaldo Bellomo, Suvi Vaara, Laurent Bitker, Glenn Eastwood. Canberra Hospital: Frank van Haren, Liam Byrne, Mary Nourse, Samantha Adam, Clare Robertson, Josie Russell-Brown, Shakira Spiller. Ghent University Hospital: Eric Hoste, Jan Fierens, Pieter Nepuydt, Daisy Vermeiren, Ingrid Herck, Druwe Patrick, Luc De Crop, Stephanie Bracke. Guys and St Thomas' Hospital: Marlies Ostermann, Andy Retter, Sara Campos, Gill Arbane, Andrea Kelly, Neus Grau Novellas, Rosario Lim, Martina Marotti, Aneta Bociek, Tim Jones, Christopher Whitton, Andrew Slack, Luigi Camporota, Simon Sparkes, Duncan Wyncoll. Helsinki University Hospital: Suvi Vaara, Minna Bäcklund, Ville Pettilä, Jonna Heinonen, Leena Pettilä, Sari Sutinen, Elina Lappi. Lausanne University Hospital Antoine Schneider, Elettra Poli, Marco Altarelli, Michel Thibault, Philippe Eckert, Madeleine Schnorf. Royal London Hospital: John Prowle, Ryan Haines, Richard Cashmore, Alex Fowler, Filipa Dos Santos, Amaia Garcia, Maria Fernandez, Tim Martin, Ruzena Uddin.

\section{Funding}

Open access funding provided by University of Helsinki including Helsinki University Central Hospital. The trial has received support from the Academy of Finland (317061), Orion Research Foundation, and state funding for universitylevel health research (TYH2017241). STV has received a Fellowship grant from the Sigrid Juselius Foundation. In the UK, the study was supported by an investigator-initiated research grant from Fresenius Medical Care. The funders had no role in the trial design, conduction, or interpretation of the results.

\section{Data availability}

\section{A dataset is available as supplemental information.}

\section{Declarartions}

\section{Conflicts of interest}

The authors declare no conflict of interest.

\section{Open Access}

This article is licensed under a Creative Commons Attribution-NonCommercial 4.0 International License, which permits any non-commercial use, sharing, adaptation, distribution and reproduction in any medium or format, as long as you give appropriate credit to the original author(s) and the source, provide a link to the Creative Commons licence, and indicate if changes were made. The images or other third party material in this article are included in the article's Creative Commons licence, unless indicated otherwise in a credit line to the material. If material is not included in the article's Creative Commons licence and your intended use is not permitted by statutory regulation or exceeds the permitted use, you will need to obtain permission directly from the copyright holder. To view a copy of this licence, visit http://creativecommons.org/licen ses/by-nc/4.0/.

\section{Publisher's Note}

Springer Nature remains neutral with regard to jurisdictional claims in published maps and institutional affiliations.

Received: 26 February 2021 Accepted: 1 April 2021

Published online: 7 May 2021

References

1. Nisula S, Kaukonen KM, Vaara ST, Korhonen AM, Poukkanen M, Karlsson S et al (2013) Incidence, risk factors and 90-day mortality of patients with 
acute kidney injury in Finnish intensive care units: the FINNAKI study. Intensive Care Med 39:420-428

2. Hoste EA, Bagshaw SM, Bellomo R, Cely CM, Colman R, Cruz DN et al (2015) Epidemiology of acute kidney injury in critically ill patients: the multinational AKI-EPI study. Intensive Care Med 41:1411-1423

3. KDIGO (2012) KDIGO clinical practice guideline for acute kidney injury. Kidney Inter Suppl 1-138

4. Meersch M, Schmidt C, Hoffmeier A, Van Aken H, Wempe C, Gerss J et al (2017) Prevention of cardiac surgery-associated AKI by implementing the KDIGO guidelines in high risk patients identified by biomarkers: the PrevAKI randomized controlled trial. Intensive Care Med 43:1551-1561

5. Pickkers P, Mehta RL, Murray PT, Joannidis M, Molitoris BA, Kellum JA et al (2018) Effect of human recombinant alkaline phosphatase on 7-day creatinine clearance in patients with sepsis-associated acute kidney injury: a randomized clinical trial. JAMA 320:1998-2009

6. Prowle JR, Kirwan CJ, Bellomo R (2014) Fluid management for the prevention and attenuation of acute kidney injury. Nat Rev Nephrol 10:37-47

7. RENAL Replacement Therapy Study Investigators (2012) An observational study fluid balance and patient outcomes in the randomized evaluation of normal vs. augmented level of replacement therapy trial* . Crit Care Med 40:1753-1760

8. Vaara ST, Korhonen AM, Kaukonen KM, Nisula S, Inkinen O, Hoppu S et al (2012) Fluid overload is associated with an increased risk for 90-day mortality in critically ill patients with renal replacement therapy: data from the prospective FINNAKI study. Crit Care 16:R197

9. Grams ME, Estrella MM, Coresh J, Brower RG, Liu KD (2011) Fluid balance, diuretic use, and mortality in acute kidney injury. Clin J Am Soc Nephrol 6:966-973

10. Bouchard J, Soroko SB, Chertow GM, Himmelfarb J, Ikizler TA, Paganini EP et al (2009) Fluid accumulation, survival and recovery of kidney function in critically ill patients with acute kidney injury. Kidney Int 76:422-427

11. Messmer AS, Zingg C, Müller M, Gerber JL, Schefold JC, Pfortmueller CA (2020) Fluid overload and mortality in adult critical care patients-a systematic review and meta-analysis of observational studies. Crit Care Med 48:1862-1870

12. Raimundo M, Crichton S, Martin JR, Syed Y, Varrier M, Wyncoll D et al (2015) Increased fluid administration after early acute kidney injury is associated with less renal recovery. Shock 44:431-437

13. Zhang J, Crichton S, Dixon A, Seylanova N, Peng ZY, Ostermann M (2019) Cumulative fluid accumulation is associated with the development of acute kidney injury and non-recovery of renal function: a retrospective analysis. Crit Care 23:392

14. Hjortrup PB, Haase N, Bundgaard H, Thomsen SL, Winding R, Pettila V et al (2016) Restricting volumes of resuscitation fluid in adults with septic shock after initial management: the CLASSIC randomised, parallel-group, multicentre feasibility trial. Intensive Care Med 42:1695-1705

15. Martensson J, Bihari S, Bannard-Smith J, Glassford NJ, Lloyd-Donald P, Cioccari L et al (2018) Small volume resuscitation with 20\% albumin in intensive care: physiological effects: the SWIPE randomised clinical trial. Intensive Care Med 44:1797-1806

16. Chen C, Kollef MH (2015) Targeted fluid minimization following initial resuscitation in septic shock: a pilot study. Chest 148:1462-1469

17. Wiedemann HP, Wheeler AP, Bernard GR, Thompson BT, Hayden D, deBoisblanc B et al (2006) Comparison of two fluid-management strategies in acute lung injury. N Engl J Med 354:2564-2575

18. Myles PS, Bellomo R, Corcoran T, Forbes A, Peyton P, Story D et al (2018) Restrictive versus liberal fluid therapy for major abdominal surgery. $\mathrm{N}$ Engl J Med 378:2263-2274

19. Pickkers P, Ostermann M, Joannidis M, Zarbock A, Hoste E, Bellomo R et al (2017) The intensive care medicine agenda on acute kidney injury. Intensive Care Med 43:1198-1209
20. Ostermann M, Bellomo R, Burdmann EA, Doi K, Endre ZH, Goldstein SL et al (2020) Controversies in acute kidney injury: conclusions from a Kidney Disease: Improving Global Outcomes (KDIGO) conference. Kidney Int 98:294-309

21. Vaara ST, Ostermann M, Selander T, Bitker L, Schneider A, Poli E et al (2020) Protocol and statistical analysis plan for the restricted fluid therapy versus standard treatment in acute kidney injury-REVERSE-AKI randomized controlled pilot trial. Acta Anaesthesiol Scand 64:831-838

22. Kahan BC, Morris TP (2012) Reporting and analysis of trials using stratified randomisation in leading medical journals: review and reanalysis. BMJ 345:e5840

23. Grissom CK, Hirshberg EL, Dickerson JB, Brown SM, Lanspa MJ, Liu KD et al (2015) Fluid management with a simplified conservative protocol for the acute respiratory distress syndrome*. Crit Care Med 43:288-295

24. Semler MW, Janz DR, Casey JD, SelfWH, Rice TW (2020) Conservative fluid management after sepsis resuscitation: a pilot randomized trial. $\rfloor$ Intensive Care Med 35:1374-1382

25. Meyhoff TS, Hjortrup PB, Moller MH, Wetterslev J, Lange T, Kjaer MN et al (2019) Conservative vs liberal fluid therapy in septic shock (CLASSIC) trial-protocol and statistical analysis plan. Acta Anaesthesiol Scand 63:1262-1271

26. Self WH, Semler MW, Bellomo R, Brown SM, deBoisblanc BP, Exline MC et al (2018) Liberal versus restrictive intravenous fluid therapy for early septic shock: rationale for a randomized trial. Ann Emerg Med 72:457-466

27. Macdonald SPJ, Keijzers G, Taylor DM, Kinnear F, Arendts G, Fatovich DM et al (2018) Restricted fluid resuscitation in suspected sepsis associated hypotension (REFRESH): a pilot randomised controlled trial. Intensive Care Med 44:2070-2078

28. Corl KA, Prodromou M, Merchant RC, Gareen I, Marks S, Banerjee D et al (2019) The restrictive IV fluid trial in severe sepsis and septic shock (RIFTS): a randomized pilot study. Crit Care Med 47:951-959

29. Berthelsen RE, Perner A, Jensen AK, Rasmussen BS, Jensen JU, Wiis J et al (2018) Forced fluid removal in intensive care patients with acute kidney injury: the randomised FFAKI feasibility trial. Acta Anaesthesiol Scand 62:936-944

30. Silversides JA, Fitzgerald E, Manickavasagam US, Lapinsky SE, Nisenbaum $R$, Hemmings $N$ et al (2018) Deresuscitation of patients with iatrogenic fluid overload is associated with reduced mortality in critical illness. Crit Care Med 46:1600-1607

31. Van Regenmortel $N$, Verbrugghe $W$, Roelant $E$, Van den Wyngaert $T$, Jorens PG (2018) Maintenance fluid therapy and fluid creep impose more significant fluid, sodium, and chloride burdens than resuscitation fluids in critically ill patients: a retrospective study in a tertiary mixed ICU population. Intensive Care Med 44:409-417

32. Lindén-Sønders $\varnothing A$, Jungner $M$, Spångfors $M$, Jan M, Oscarson A, Choi S et al (2019) Survey of non-resuscitation fluids administered during septic shock: a multicenter prospective observational study. Ann Intensive Care 9:132

33. Agrinier N, Monnier A, Argaud L, Bemer M, Virion JM, Alleyrat C et al (2019) Effect of fluid balance control in critically ill patients: design of the stepped wedge trial POINCARE-2. Contemp Clin Trials 83:109-116

34. Douglas IS, Alapat PM, Corl KA, Exline MC, Forni LG, Holder AL et al (2020) Fluid response evaluation in sepsis hypotension and shock: a randomized clinical trial. Chest 158:1431-1445

35. Vignon P, Evrard B, Asfar P, Busana M, Calfee CS, Coppola S et al (2020) Fluid administration and monitoring in ARDS: which management? Intensive Care Med 46:2252-2264 